\title{
Visual prior entry for foreground figures
}

\author{
Benjamin D. Lester, Lauren N. Hecht, and Shaun P. Vecera \\ University of Iowa, Iowa City, Iowa
}

\begin{abstract}
Attended stimuli reach perceptual-level processes before unattended stimuli do, a finding that is referred to as visual prior entry. We asked whether a similar effect arises for salient objects (foreground figures) in a visual scene. If prior entry holds for figure-ground perception, targets will be perceived to appear earlier on figures than on grounds. Participants performed a temporal order judgment by reporting the order in which targets appeared. Participants perceived that targets appearing on foreground figures occurred earlier than did those appearing on backgrounds. These findings did not result from a response bias for targets appearing on figures. Most important, when figures and grounds were spatially separated and did not share an edge, no prior-entry effects were observed. Our results suggest that figural regions are available to perceptual-level processes sooner than are grounds.
\end{abstract}

Visual scenes present an observer with a wealth of information. Because of scene complexity, however, relevant information can be difficult to extract. The visual system can reduce this complexity and isolate information by labeling some regions as foreground figures (i.e., candidate objects) and others as backgrounds (i.e., space between objects). This figure-ground assignment allows resourcelimited processes, such as attention, action, or memory, to be directed toward figures, not grounds. There are many cues for figure-ground assignment, including image cues such as area, symmetry, convexity, or lower region (see Palmer, 1999, 2002), and higher-level cues such as object familiarity or attention (Driver \& Baylis, 1996; Peterson, 1994; Vecera, Flevaris, \& Filapek, 2004).

Although there are many known cues to figure-ground assignment, much less is known about the consequences, or effects, of figure-ground assignment. Phenomenologically, figures appear to be more shape-like and more salient than grounds (Rubin, 1915/1958). One possible source for the increased perceptibility of figures over grounds is that figures might be processed faster than grounds are, allowing figures to become available for perceptual processing earlier than grounds are. Numerous studies of visual attention have suggested that attended events are perceived before unattended events, a finding that has been termed the doctrine of prior entry (Shore \& Spence, 2005; Titchener, 1908). The present experiments asked whether nonattentional factors, such as scene properties that affect figural status, produce prior-entry-like effects. In short, do some portions of scenes (figures) receive perceptual processing ahead of others (grounds)?

Prior entry is typically studied using temporal-orderjudgment (TOJ) tasks, in which participants report which of two targets appeared first. The delay between the targets' onsets varies (the stimulus onset asynchrony, SOA), and attention is directed to one of the targets before it appears. Targets at attended locations are perceived to occur earlier than targets at unattended locations do. Although early studies of prior entry were subject to alternative explanations such as response bias (see Pashler, 1998), more recent studies have ruled out response biases. For example, Shore, Spence, and Klein (2001) had participants report a target dimension that was orthogonal to the direction of attention, and they included a condition in which participants reported which stimulus appeared second. Prior-entry effects have continued to be observed under these more stringent conditions, suggesting that attention speeds perception (Shore et al., 2001).

To determine whether a prior entry effect exists for figure-ground processes, we followed previous work and used a TOJ task that eliminated response biases. Participants viewed displays containing two regions (Figure 1); two targets, an $\mathrm{x}$ and an $\mathrm{o}$, then appeared, one on each region. The interval between the targets' onset varied, and participants reported either which target appeared first or which appeared second. Any bias in responding first to targets on figures was eliminated when participants reported which target had appeared second (Shore et al., 2001). This procedure allowed us to compute the point of subjective simultaneity (PSS) for temporal discrimination. The PSS reflects the time at which participants discriminate the temporal onsets with 50\% accuracy; that is, the PSS is the point at which participants perceive the appearance of the stimuli to be simultaneous. We computed the percentage of the time that the target ( $\mathrm{x}$ or o) was judged to occur first or second. If the target on the figure was perceived to occur earlier than the target on the ground did, the target on the ground would need to appear before that on the figure for the two targets to be perceived to occur at the same time. That is, if targets on figures are perceived to

S.P.Vecera, shaun-vecera@uiowa.edu 
A

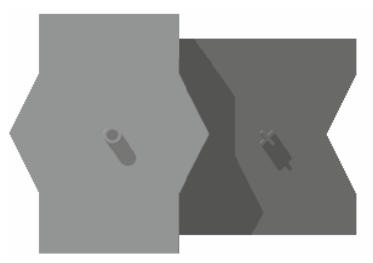

B
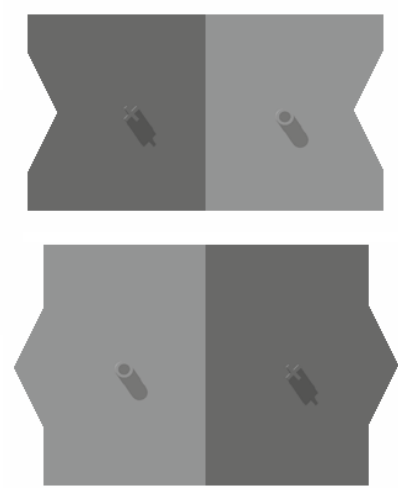

Figure 1. Sample stimuli that were used in the experiments. (A) Figure-ground display in which the symmetric convex region (depicted in light gray) appeared as the foreground region. (B) Two types of ambiguous displays that did not produce a strong figure-ground segregation.

occur earlier than those on grounds do, the PSS should be significantly different from zero.

\section{EXPERIMENT 1}

\section{Method}

Participants. Thirty-two University of Iowa undergraduates with normal or corrected-to-normal vision volunteered for course credit. The report order (which target appeared first or which appeared second) was manipulated as a between-subjects variable, and 16 volunteers participated in each of these target-report conditions.

Stimuli. Figure 1 depicts the two different display types, one with a strong figure-ground assignment (Figure 1A) and one with an ambiguous figure-ground assignment (Figure 1B). Each display contained a green region (RGB $0,132,0$ ) and a red-orange region (RGB 238,83,15). Each color occurred with equal frequency on the left and right regions. Displays appeared against a black background.

In figure-ground displays, the symmetric convex figure subtended approximately $3.73^{\circ} \times 4.60^{\circ}$ of visual angle; the concave shaded ground region subtended approximately $3.34^{\circ} \times 3.73^{\circ}$. Both regions were equally likely to appear on either side of fixation. There were two types of ambiguous displays, one with two convex regions and one with two concave regions. Each region in a convex display measured $3.58^{\circ} \times 4.60^{\circ}$, and each region in a concave display measured $3.42^{\circ} \times 3.80^{\circ}$. The ambiguous displays lacked the depth cues that were present in the figure-ground displays, thereby eliminating strong figure-ground assignment.

Two targets were presented in each display, with one target appearing on each shape. The targets were a small $\mathrm{x}$ and a small o that both subtended approximately $0.40^{\circ} \times 0.40^{\circ}$ of visual angle. The targets were the same color as the region on which they were presented, thereby eliminating the appearance that the targets were superimposed over the displays. Across trials, each target appeared with equal frequency on each region of the display and appeared $1.8^{\circ}$ from fixation.

Procedure. Each trial began with a 500-msec fixation point that participants were instructed to fixate throughout each trial. The fixation point appeared on the contour between the two regions. A figure-ground display was then presented for $500 \mathrm{msec}$, followed by the two targets, which were presented at differing onsets. The targets appeared to grow out of the middle of the displays and then recede as a result of our varying the lengths of shadows that were cast by the targets. The targets grew and receded across $225 \mathrm{msec}$. One target appeared and started its movement before the second target did; the second target appeared and began moving with SOAs of 26, 50, 100, and $150 \mathrm{msec}$; SOAs were randomized throughout the experiment. The target on the figure moved ahead of that on the ground in half of the trials and behind that on the ground in the other half of the trials. Negative SOAs corresponded to targets on the figure moving first; positive SOAs corresponded to targets on the ground moving first.

Half of the participants judged which target ( $\mathrm{x}$ or o) appeared to move first, and half judged which target appeared to move second. Participants performed 512 trials and responded via keypress.

\section{Results and Discussion}

The results appear in Figures 2A (Which moved first?) and $2 \mathrm{~B}$ (Which moved second?), which depict the proportion of the times that the target on the ground was judged as moving either first or second, respectively, averaged across all participants. The curves that are shown in Figure 2 are the best-fitting logistic functions fitted to the average data. All statistical analyses, however, were based on the best-fitting logistic curves fitted to each participant's data. The curve fits for individual observers produced $R^{2}$ values of .95 or higher.

As is evident from Figures 2A and 2B, the percentage of trials in which the ground was perceived as moving first increased as SOA increased. More importantly, however, is the rightward shift in the PSS for the figure-ground stimuli compared with the PSS for the ambiguous stimuli: Participants required the target to move earlier on the ground in order for them to be able to perceive the targets as moving simultaneously. This pattern of results was observed in a majority (70\%) of participants.

To quantify these observations, we calculated a PSS for each observer on the basis of that observer's best-fitting logistic function; the PSS was computed as the point at which the observer was equally likely to report seeing each target as moving first. An initial, combined analysis of the accuracy of reports from the "which first?" and "which second?" conditions revealed less accurate responses in the "which first?" condition than the "which second?" condition (79.5\% vs. $83.7 \%)$. Task did not interact with the remaining factors, however, so we collapsed across task when analyzing the PSSs. We also found that the two types of ambiguous displays, convex and concave, produced similar results, so we averaged performance across these displays.

Analysis of the PSSs revealed a PSS of $9.5 \mathrm{msec}$ for the figure-ground displays, which differed significantly from zero $[t(31)=3.20, p<.005]$. This positive PSS indicates that targets on grounds needed to occur, on average, $9.5 \mathrm{msec}$ ahead of those on figures in order for the two targets to be perceived to occur simultaneously. For ambiguous displays, the PSS was $-2.6 \mathrm{msec}$, which did not differ significantly from zero $[t(31)=1.24, p>.20]$, indicating that the targets in neither region in these displays had a temporal processing advantage over the targets in the other region. The PSS for the figure-ground displays was significantly larger than the PSS for the ambiguous displays $[t(31)=3.63, p<.001]$.

The present findings support a prior-entry effect of figure-ground assignment. Targets that were presented on figures were perceived to occur earlier than targets appearing on grounds or on ambiguous regions. For the two 


\section{A Experiment 1: “Which First?”}

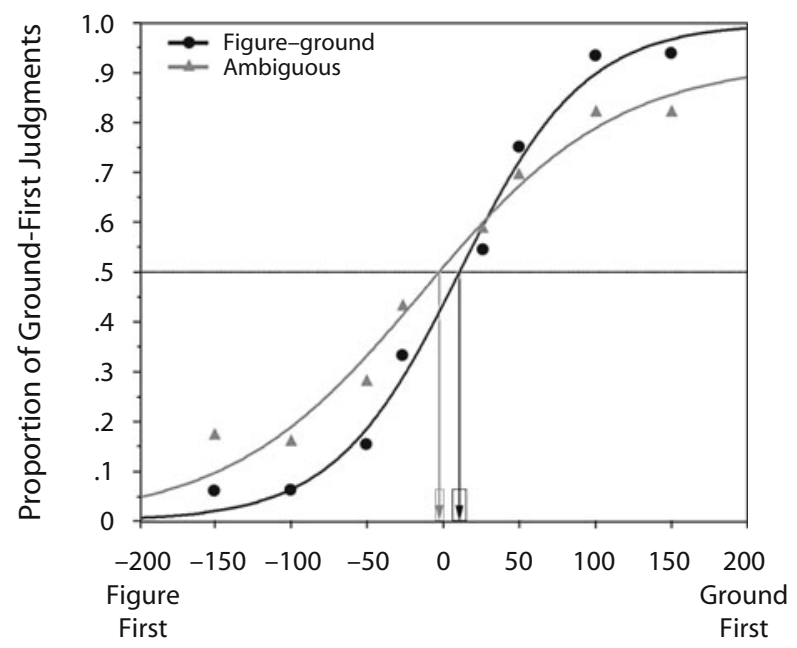

SOA (msec)

\section{B Experiment 1: “Which Second?”}

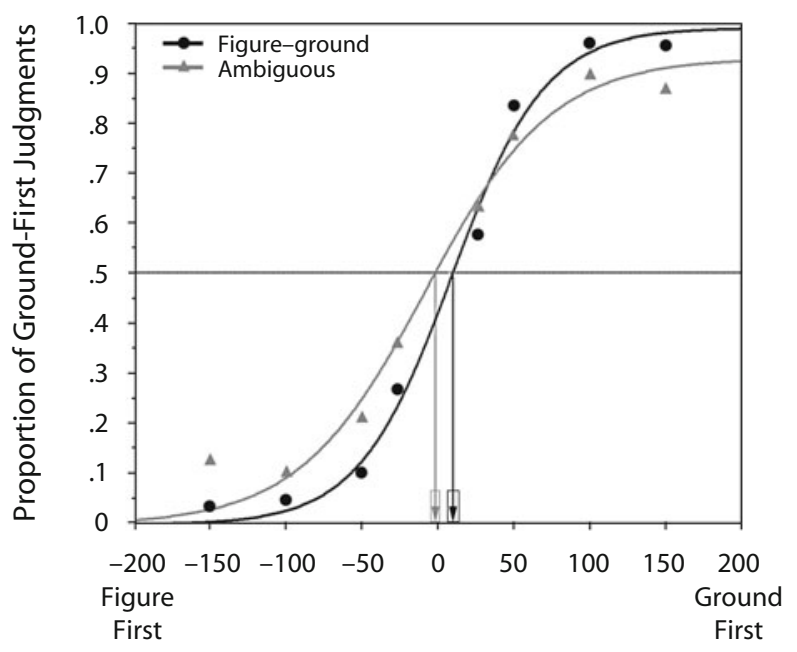

SOA (msec)

\section{Experiment 2: Separated Regions}

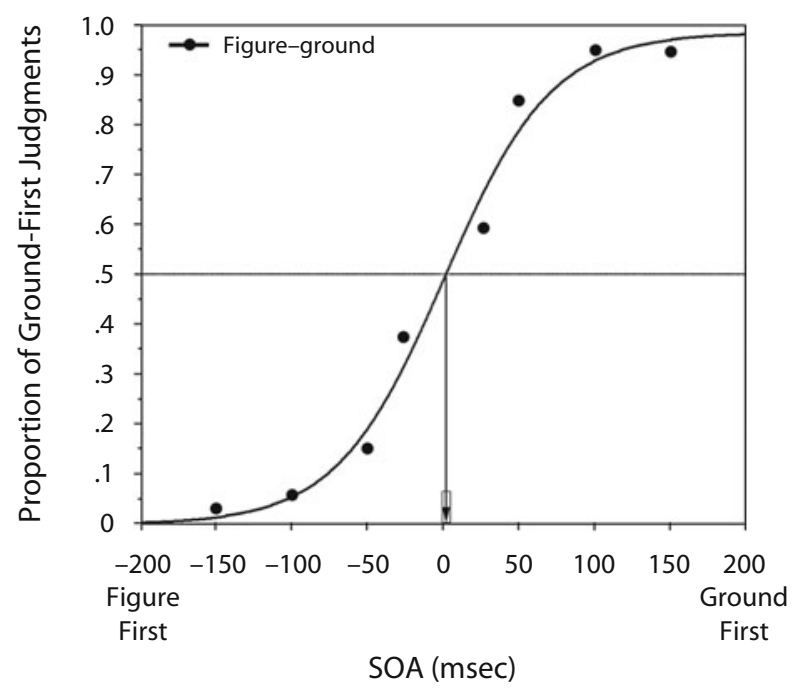

Figure 2. Results from Experiments 1 and 2. (A) Results from Experiment 1, in which participants judged the target that they perceived to move first, and (B) results from Experiment 1, in which participants judged the target that they perceived to move second. The figure-ground displays exhibited a rightward shift in the PSS (black arrow) when compared with ambiguous displays, in which either region could be perceived as figure (gray arrow). No such figural advantage appeared in (C) the results from Experiment 2, in which the regions were separated spatially. The graphs plot the proportion of trials in which the target on the ground was judged to occur first as a function of SOA. Negative SOAs are for targets on figures that appeared first, and positive SOAs are for targets on grounds that appeared first. All error bars are within-subjects $95 \%$ confidence intervals.

targets to be perceived to occur simultaneously, targets on the ground needed to onset earlier than those on the figure. Importantly, our procedures ruled out biases to respond first to figures, because we continued to find a figural advantage when participants reported which target appeared second.

Although the results of Experiment 1 were straightforward, there are two issues to discuss. One concern is that the figure-ground displays were visible for $500 \mathrm{msec}$ before the targets appeared to allow the regions to be fully segregated, and participants might have made eye movements and fixated one of the regions, allowing targets on figures to be processed more quickly than were those on grounds. Preferential eye fixation of one of the regions is not a straightforward alternative to our results, however, because in temporal perception, targets in the periphery (i.e., more distant from fixation) are processed faster than are central targets (see, e.g., Carrasco, McElree, Denisova, $\&$ Giordano, 2003). Although there are numerous differences between the present procedure and Carrasco et al.'s, 
their results would suggest that for our results to show a temporal advantage for figures, the ground region- not the figural region - would need to be fixated. Such a preferential eye fixation of the ground is problematic, because the eyes typically are directed toward objects (i.e., figures). Nevertheless, to address this concern, we tested an additional 7 participants in the "which first?" task, monitoring their eye position and excluding trials in which fixation was not maintained (less than $3 \%$ of trials). The results from this control study were similar to those from Experiment 1 , with a PSS on figure-ground displays of $8.8 \mathrm{msec}$, indicating that targets on the ground needed to lead those on the figure by an average of $8.8 \mathrm{msec}$ for the two to be perceived as simultaneous. For ambiguous displays, there was no systematic shift in the PSS; these displays produced a PSS of $-2.3 \mathrm{msec}$. These PSS values did not differ significantly from those that were observed in Experiment 1 $[t(37)<1$ for the figure-ground displays; $t(37)<1$ for the ambiguous displays]. Also, when eye position was monitored, the difference in PSSs between the figure-ground and ambiguous conditions approached significance $[t(6)=$ $1.63, p=.07$ (one-tailed)]. Thus, our findings from Experiment 1 do not seem to have been caused by participants preferentially fixating either region.

A second issue for discussion is response bias. Our procedure carefully followed previous work in ruling out response bias; participants responded to target features that were orthogonal to figure-ground assignment, and we controlled for the order of report across the conditions in Experiment 1. One might express the concern, however, that with figure-ground displays a response bias is more complex. Specifically, the response bias might be to respond first to targets on figures and second (or, more generally, later) to targets on grounds. There are no data to support this more complex response bias, however; response bias for TOJs following figure-ground manipulations need not be different from that for TOJs following attentional manipulations. The "which first?" and "which second?" control procedure, moreover, produced more reliable results than did other attempts to rule out response bias. For example, one possible procedure to rule out such a sophisticated response bias is to include a response alternative of "simultaneous" for participants; such a response alternative would reduce a response bias by freeing participants from responding to the target on the figure when the SOAs were short and participants were uncertain about which response to make (for discussion, see Stelmach \& Herdman, 1991). The results of previous studies that provided a "simultaneous" response, however, suggest that this response has been chosen inconsistently across studies. For example, Stelmach and Herdman's participants used a "simultaneous" response infrequently (less than $5 \%$ of trials), whereas Jaskowski's (1993) participants used this response alternative on the majority of trials. On the basis of the results in the literature and in our own pilot work using a "simultaneous" response, we concluded that adding such a response might not conclusively rule out response bias.

A third issue concerns an alternative interpretation of our results: Attention could be attracted more to regions that contain figural cues (e.g., convexity, symmetry) than to regions that lack these cues; thus, our findings could be produced by attention, not figure-ground assignment per se. To address this alternative, in Experiment 2 participants performed the TOJ task that was used in Experiment 1 but with figure-ground displays that separated the two regions. Separating the regions eliminated their competition for figural status and assignment of the shared edge to the figure. If our previous results were produced by attention to convex, symmetric regions only-not to figure-ground assignment - separating the regions would have no effect. Attention would be drawn to convex, symmetric regions ("figures" in Experiment 1), and targets on such regions would be perceived to occur earlier than targets on other regions do. In contrast, if our results depended on figure-ground assignment itself, there would be no differences in temporal perception between targets on the two regions, because the regions would not compete for the assignment of a shared edge.

\section{EXPERIMENT 2}

\section{Method}

Participants. Sixteen University of Iowa undergraduates with normal or corrected vision volunteered for course credit.

Stimuli and Procedure. The stimuli and procedure were identical to those in Experiment 1, with the following exceptions. First, participants only viewed figure-ground displays; ambiguous displays were not presented. Second, the regions in these displays were separated by $0.5^{\circ}$ of visual angle and did not share an edge. To compensate for the fact that the targets appeared further in the periphery than did those in Experiment 1, the targets in the present experiment were increased in size in accordance with the cortical magnification factor (Rovamo \& Virsu, 1979) that was suggested by Wolfe, O'Neill, and Bennett (1998) for discriminations tasks. The scaled targets measured $0.50^{\circ} \times 0.50^{\circ}$.

\section{Results and Discussion}

The data were treated in the same manner as were those in Experiment 1. The results for Experiment 2 appear in Figure 2C. Analysis of the PSS that was computed from the fitted logistic functions revealed a PSS of $-0.53 \mathrm{msec}$, which did not differ significantly from zero $[t(15)<1]$. Moreover, the magnitude of the PSS in Experiment 2 was significantly smaller than that of the PSS from the figureground displays in Experiment $1[t(46)=2.14, p<.05]$. These findings suggest that competition for figural status, which is increased by having two regions share a contour, was important in determining temporal perception differences between the two regions.

The present results indicate that the prior-entry-like effect that was observed in Experiment 1 depends critically on the assignment of one region as a foreground figure. When regions do not share an edge and do not compete for figural status, temporal perception does not differ significantly between the two regions. The separation between the regions in Experiment 2 likely prevented the convex region (figure) from speeding perceptual processes consistently on most trials. The present findings indicate that figural status, in a manner similar to that of attentional cuing, affects the time at which targets are available for perceptual processing. 
Although the results of the present experiments strongly support a prior-entry-effect for foreground figures, this conclusion depends on an assumption that participants assigned figural status to the convex region that shared a contour with a concave region, as was the case in Experiment 1, but did not assign figural status to the same region when it was separated from another region, as was the case in Experiment 2. To ensure that this assumed figure-ground assignment (or lack thereof) did result from our displays, we conducted a control experiment. In Experiment 3, participants performed a targetdiscrimination task in which a single target appeared on a region and participants reported whether the target was an x or an o. Nelson and Palmer (2007) reported that such a discrimination task is sensitive to figure-ground assignment; their participants were faster to discriminate targets that appeared on figures than they were to discriminate those that appeared on grounds (see also Lazareva, Castro, Vecera, \& Wasserman, 2006). If our displays produce a figure-ground assignment that is consistent with the results of Experiments 1 and 2, we would expect targets to be discriminated fastest on figures that are in figure-ground displays in which the regions share an edge and compete for edge assignment. Targets on other regions should be discriminated relatively slowly because of the absence of a strong figureground interpretation.

\section{EXPERIMENT 3}

\section{Method}

Participants. Ten University of Iowa undergraduates with normal or corrected vision volunteered for course credit.

Stimuli and Procedure. The stimuli were the three display types that were used in Experiments 1 and 2-namely, figure-ground displays that shared a contour, ambiguous displays, and the separated displays from Experiment 2. The procedure involved presenting a single target on one of the two regions in a display. Participants were instructed to report whether the target was an $\mathrm{x}$ or an o as quickly and accurately as possible. Following a $500-\mathrm{msec}$ fixation point, a display with a single target that had already grown out to its furthest position appeared for $200 \mathrm{msec}$. The display then disappeared while participants responded.

\section{Results and Discussion}

Reaction times (RTs) longer than 2,000 msec and shorter than $150 \mathrm{msec}$ were excluded from the analyses; this trimming removed less than $5 \%$ of the data. The mean RTs appear in Table 1. The results indicated that targets on a figure were discriminated faster than were those on a ground in only those displays in which the two regions shared a contour. We analyzed the RTs with a two-factor ANOVA, with display type (figure-ground, ambiguous, or separated) and target location (figure or ground) as factors. ("Figure" and "ground" were chosen arbitrarily for ambiguous displays; for separated displays, the figures were convex regions and grounds were concave regions.) We conducted planned comparisons on the RT for figures and grounds for each of the three display types.

Our analysis revealed neither a main effect for display type $[F(2,18)<1$, n.s. $]$ nor a main effect for target location $[F(1,9)<1$, n.s. $]$. There was a significant interaction $[F(2,18)=4.54, p<.05]$, suggesting that the target location depended on the display type. The planned comparisons indicated that this was indeed the case. Figures were discriminated faster than were grounds when the two regions shared a contour $[t(9)=2.32, p<.05]$, but not when the regions were part of an ambiguous display $[t(9)=2.03, p>.05]$, or when the regions were separated $[t(9)<1$, n.s.]. Accuracy was high $(>94 \%$ in all conditions) and showed no statistically significant effects between targets on figures and those on grounds.

Consistent with previous findings in the literature (e.g., Lazareva et al., 2006; Nelson \& Palmer, 2007), target discrimination was faster when targets appeared on a figure than when they appeared on a ground, indicating a processing preference for figures over grounds. Importantly, this same figural advantage did not appear for our ambiguous stimuli or for separated regions. These findings verify that participants assigned figural status to the convex regions in our display, but only when those regions shared a contour with another region, a conclusion that is consistent with the figure-ground effects that are based on TOJs from Experiments 1 and 2.

\section{GENERAL DISCUSSION}

Our experiments demonstrate a prior-entry-like effect for figure-ground assignment in which targets appearing on figures are perceived to occur earlier than targets appearing on nonfigural regions do. Our findings are likely not due to a response bias favoring targets on figures, because temporal processing remained better for figures than for grounds when participants reported which of two targets appeared second. Most important, our results depended on figural assignment: When regions are separated and do not share an edge, no differences in temporal perception appeared between the regions.

Table 1

Mean RTs and Accuracy for Perceptual-Discrimination Task (Experiment 3)

\begin{tabular}{|c|c|c|c|c|c|c|c|c|}
\hline \multirow[b]{3}{*}{ Display Type } & \multicolumn{4}{|c|}{ Target on Figure } & \multicolumn{4}{|c|}{ Target on Ground } \\
\hline & \multicolumn{2}{|c|}{ RT } & \multicolumn{2}{|c|}{ Accuracy } & \multicolumn{2}{|c|}{ RT } & \multicolumn{2}{|c|}{ Accuracy } \\
\hline & $M$ & $\mathrm{CI}$ & $\%$ & $\mathrm{CI}$ & $M$ & $\mathrm{CI}$ & $\%$ & $\mathrm{CI}$ \\
\hline Shared contour & 581.0 & 14.9 & 94.4 & 1.8 & 611.5 & 14.9 & 96.7 & 1.8 \\
\hline Ambiguous & 597.7 & 9.8 & 94.8 & 1.0 & 580.2 & 9.8 & 95.4 & 1.0 \\
\hline Separated contour & 598.8 & 9.3 & 95.8 & 1.5 & 597.0 & 9.3 & 97.3 & 1.5 \\
\hline
\end{tabular}

Note-CI, $95 \%$ confidence intervals on the figure-versus-ground comparisons for each display type. 
Beyond the present results, there might be other temporal consequences of figure-ground assignment. For example, temporal information influences figure-ground assignment: Regions that flicker at higher temporal frequencies tend to be perceived as grounds, and those that flicker at lower frequencies tend to be perceived as figures (Klymenko \& Weisstein, 1989). Also, figures may hold perceptual processes longer than do grounds. Such an effect could appear in a flicker fusion task, in which two targets are presented in rapid succession at the same location. If figures hold processing longer than do grounds, then figures might have higher flicker fusion thresholds than do grounds (cf. Yeshurun \& Levy, 2003); similarly, if figures hold processing longer than grounds, then detection of the offset of a target might be slower for figures than for grounds (cf. Rolke, Ulrich, \& Bausenhart, 2006).

The present study is one of an increasing number of studies that have found that figure-ground processes show effects that are similar to the effects of attention. In addition to our demonstration of the temporal-perception advantages of figures, others have shown that visual targets appearing on figures are discriminated faster and more accurately than are those on grounds (Lazareva et al., 2006; Nelson \& Palmer, 2007; Wong \& Weisstein, 1982). Although these latter studies have reported an RT difference between targets on figures and those on grounds, the cause of this figural advantage is not known. The figural advantage could be the result of better spatial perception of targets on figures than on grounds, or of improved temporal perception of targets on figures versus grounds. Our results point to a specific source of the figural advantage: Figures may alter temporal perception, allowing targets on figures to be perceived to occur earlier than those on grounds do. In a speeded discrimination task (see, e.g., Nelson \& Palmer, 2007), this change in temporal perception might produce faster RTs to targets on figures than to those on grounds.

Importantly, our results also demonstrate that competition among regions is critical to inducing processing differences between figures and grounds. Separated regions do not produce the same perceptual consequences as does figure-ground assignment. This latter finding suggests that attention to a region may not fully explain the perceptual differences between figures and grounds that we have reported. Instead, as recent neurophysiological findings have suggested, attention might depend on figure-ground assignment; attentional effects are stronger when attention is directed to a figure than when it is directed to a ground (see Qiu, Sugihara, \& von der Heydt, 2007). Thus, figureground assignment and spatial attention may be highly interactive and produce similar effects, although they can remain distinct, dissociable processes (see, e.g., Kimchi \& Peterson, 2008; Vecera \& Behrmann, 1997).

\section{AUTHOR NOTE}

This research was supported in part by NSF Grant BCS 03-39171 to S.P.V. Correspondence should be addressed to S. P. Vecera, Department of Psychology, E11 Seashore Hall, University of Iowa, Iowa City, IA 52242-1407 (e-mail: shaun-vecera@uiowa.edu).

\section{REFERENCES}

Carrasco, M., McElree, B., Denisova, K., \& Giordano, A. M. (2003). Speed of visual processing increases with eccentricity. Nature Neuroscience, 6, 699-700.

Driver, J., \& BAYLIS, G. C. (1996). Edge-assignment and figure-ground segmentation in short-term visual matching. Cognitive Psychology, 31, 248-306.

JASKOWSKI, P. (1993). Selective attention and temporal-order judgment. Perception, 22, 681-689.

Kimchi, R., \& Peterson, M. A. (2008). Figure-ground segmentation can occur without attention. Psychological Science, 19, 660-668.

Klymenko, V., \& Weisstein, N. (1989). Figure and ground in space and time: 1 . Temporal response surfaces of perceptual organization. Perception, 18, 627-637.

Lazareva, O. F., Castro, L., Vecera, S. P., \& Wasserman, E. A. (2006). Figure-ground assignment in pigeons: Evidence for a figural benefit. Perception \& Psychophysics, 68, 711-724.

Nelson, R. A., \& Palmer, S. E. (2007). Familiar shapes attract attention in figure-ground displays. Perception \& Psychophysics, 69, 382-392.

Palmer, S. E. (1999). Vision science: Photons to phenomenology. Cambridge, MA: MIT Press.

Palmer, S. E. (2002). Perceptual organization in vision. In H. Pashler \& S. Yantis (Eds.), Stevens' Handbook of experimental psychology: Vol. 1. Sensation and perception (3rd ed., pp. 177-234). New York: Wiley.

Pashler, H. E. (1998). The psychology of attention. Cambridge, MA: MIT Press.

Peterson, M. A. (1994). Object recognition processes can and do operate before figure-ground organization. Current Directions in Psychological Science, 3, 105-111.

Qiu, F. T., Sugihara, T., \& VON DER Heydt, R. (2007). Figure-ground mechanisms provide structure for selective attention. Nature Neuroscience, 10, 1492-1499.

Rolke, B., Ulrich, R., \& Bausenhart, K. M. (2006). Attention delays perceived stimulus offset. Vision Research, 46, 2926-2933.

Rovamo, J., \& VIRSU, V. (1979). An estimation and application of the human cortical magnification factor. Experimental Brain Research, 37, 495-510.

RuBIN, E. (1958). Figure and ground. In D. C. Beardslee \& M. Wertheimer (Eds.), Readings in perception (pp. 194-203). Princeton, NJ: Van Nostrand. (Original work published 1915)

Shore, D. I., \& Spence, C. (2005). Prior entry. In L. Itti, G. Rees, \& J. K. Tsotsos (Eds.), Neurobiology of attention (pp. 89-95). New York: Elsevier.

Shore, D. I., Spence, C., \& Klein, R. M. (2001). Visual prior entry. Psychological Science, 12, 205-212.

Stelmach, L. B., \& Herdman, C. M. (1991). Directed attention and perception of temporal order. Journal of Experimental Psychology: Human Perception \& Performance, 17, 539-550.

TitCHENER, E. B. (1908). Lectures on the elementary psychology of feeling and attention. New York: Macmillan.

Vecera, S. P., \& Behrmann, M. (1997). Spatial attention does not require preattentive grouping. Neuropsychology, 11, 30-43.

Vecera, S. P., Flevaris, A. V., \& FilapeK, J. C. (2004). Exogenous spatial attention influences figure-ground assignment. Psychological Science, 15, 20-26.

Wolfe, J. M., O'Neill, P., \& Bennett, S. C. (1998). Why are there eccentricity effects in visual search? Visual and attentional hypotheses. Perception \& Psychophysics, 60, 140-156.

WoNG, E., \& WeISSTEIN, N. (1982). A new perceptual context-superiority effect: Line segments are more visible against a figure than against a ground. Science, 218, 587-589.

Yeshurun, Y., \& Levy, L. (2003). Transient spatial attention degrades temporal resolution. Psychological Science, 14, 225-231.

(Manuscript received March 2, 2008; revision accepted for publication March 31, 2009.) 